\title{
Concrete mindset impairs filtering in visual working memory
}

\author{
Britt Hadar $^{1} \cdot$ Roy Luria ${ }^{1,2} \cdot$ Nira Liberman ${ }^{1}$
}

Published online: 19 August 2019

(C) The Psychonomic Society, Inc. 2019

\begin{abstract}
Two studies tested whether a mindset manipulation would affect the filtering of distractors from entering visual working memory (VWM). In Study 1, participants completed a concrete mindset manipulation (by repeatedly describing how to perform an action), an abstract mindset manipulation (by repeatedly describing why to perform an action), and a baseline condition (no manipulation). In Study 2, some participants completed a concrete mindset manipulation, whereas others completed an abstract manipulation. Filtering efficiency was estimated by a change-detection task that included a condition with distractors alongside targets. We derived our prediction from construal-level theory (CLT), according to which concrete representations retain information regardless of its relevance, whereas abstract representations retain the relevant and omit the irrelevant elements of an input array. In a task that requires attending to task-relevant targets and ignoring task-irrelevant distractors in a visual array, concrete processing should impair performance relative to abstract processing. We therefore predicted that a concrete mindset would reduce filtering efficiency as compared to an abstract mindset. Consistent with our hypothesis, we found that a concrete mindset manipulation reduced filtering efficiency, as compared to both an abstract mindset manipulation (Studies 1 and 2) and the baseline condition (Study 1). These results suggest a new factor that may contribute to both individual differences and situational variation in working memory performance.
\end{abstract}

Keywords Visual working memory $\cdot$ Construal level theory $\cdot$ Abstraction $\cdot$ Filtering

Visual working memory (VWM) is a limited-capacity system that temporarily stores information to serve other, ongoing cognitive processes (Awh, Barton, \& Vogel, 2007; Baddeley, 2003; Luck \& Vogel, 2013). There are robust individual differences in VWM capacity, which covary with cognitive performance, intelligence, and academic aptitude (Alloway \& Alloway, 2010; Conway, Kane, \& Engle, 2003; Cowan et al., 2005; Fukuda, Vogel, Mayr, \& Awh, 2010; Shipstead, Redick, Hicks, \& Engle, 2012). Understanding the source of variation in VWM capacity is thus a central and important question.

One of the principal accounts of individual differences in VWM capacity attributes it to an attentional-control mechanism that prevents the storage of irrelevant or potentially distracting information (Awh \& Vogel, 2008; Conway et al.,

Nira Liberman

niralib@tauex.tau.ac.il

1 School of Psychological Sciences, Tel-Aviv University, Ramat Aviv, Tel Aviv, Israel

2 Sagol School of Neuroscience, Tel-Aviv University, Ramat Aviv, Tel Aviv, Israel
2005; Fukuda \& Vogel, 2011; Fukuda, Woodman, \& Vogel, 2015; Gaspar, Christie, Prime, Jolicœur, \& McDonald, 2016; Vogel, McCollough, \& Machizawa, 2005). When information exceeds the capacity limit, attempting to store an item beyond one's capacity is not only bound to fail, but might also interfere with already stored items. By preventing irrelevant information from entering and occupying the limited-capacity storage space in VWM, attentional control can allow more relevant information to be stored. In support of this view, Vogel, McCollough, and Machizawa showed that individuals with low VWM capacity store more irrelevant information than do individuals with high VWM capacity. In the present study, we examined the possibility that a situationally induced tendency to use concrete (vs. abstract) mental representations would reduce filtering efficiency. As we now turn to explain, we derived our prediction from the reasoning that effective filtering is a process similar to abstraction, in that both are based on a distinction between the relevant and irrelevant features of a focal stimulus.

The notion that efficient filtering is critical for effective VWM storage connects it to construal-level theory (CLT; Liberman \& Trope, 2008, 2014; Trope \& Liberman, 2010). Construal level theory views mental representations of 
information as occupying a place on a continuum that stretches between abstract and concrete. Abstract representations extract the gist of informational input and retain its most essential features, whereas concrete representations preserve a fuller array of both relevant and irrelevant features of the input. According to CLT then, the process of abstraction involves a decision, either implicit or explicit, on which attributes are central and which are peripheral, and attending to the central attributes while ignoring any peripheral attributes. For example, mentally representing a slice of pepperoni pizza as "food" involves regarding shape, color, and temperature as secondary/ irrelevant, and "can be eaten" as primary. Notably, what is central and what is peripheral may change between situations, and abstractions might change accordingly.

This definition of abstraction allows us to view visual filtering as a process that is similar to abstraction. For example, when in visual arrays triangles are defined as the targets (and remembering their color is essential for performing the task), and squares are defined as distractors (and remembering them is superfluous to performing the task), a viewer has to abstract from the input only triangles and ignore any squares. Doing so would allow him or her to store information more efficiently in VWM. More generally, filtering, just like abstraction, is a process that retains the primary, goal-relevant features of an input and eliminates the less relevant, incidental features.

\section{Study 1}

In the present study, we examined the prediction that a concrete mindset would hinder efficient filtering, whereas an abstract mindset would promote more efficient filtering. We manipulated mindset via the standard "why" versus "how" task (Freitas, Gollwitzer, \& Trope, 2004), in which participants repeatedly answer either why they would perform an activity (why maintain good physical health), thereby arriving at increasingly more abstract answers, or how they would perform the same activity, thereby arriving at increasingly more concrete answers. This manipulation has been widely used to elicit abstract versus concrete mindsets that carry over to a subsequent task (e.g., Fujita, Trope, Liberman, \& LevinSagi, 2006; Kille, Eibach, Wood, \& Holmes, 2017; Wakslak \& Trope, 2009; for a review, see Burgoon, Henderson, \& Markman, 2013; for a neurological analysis of this manipulation, see Gilead, Liberman, \& Maril, 2013). Participants completed both mindset conditions in counterbalanced order. The abstract and concrete mindset conditions were always preceded by a baseline condition (with no manipulation) to establish an initial level of filtering efficiency. Note that performance in this design might be affected by practice, fatigue or sensitization (for effects of within-participants design, see Greenwald, 1976). However, the critical comparison is between the concrete and the abstract mindset conditions, the order of which was counterbalanced between participants (for similar experimental designs, see Fujita et al., 2006; Gruber, Harvey, \& Johnson, 2009; Kross et al., 2014; Zinchenko, Conci, Müller, \& Geyer, 2018).

We measured filtering efficiency by using the changedetection task with distractors, in which participants' performance is compared between conditions that present them with visual arrays with three targets (3T), with six targets (6T), and arrays with three targets and three distractors (3T3D). Effective filtering is reflected in performing better with targets-plus-distractors arrays (3T3D) than with large 6T arrays ("filtering benefit") and not much worse than with the small $3 \mathrm{~T}$ arrays ("filtering cost").

We calculated the filtering cost as the difference in accuracy between the three-targets arrays and the filtering arrays (i.e., filtering cost $=3 \mathrm{~T}-3 \mathrm{~T} 3 \mathrm{D}$ ). Higher scores indicate that increasing the array by adding distractors that need to be filtered out interfered with performance. Filtering cost is the main measure that has been used in studies of filtering in VWM (Allon \& Luria, 2017, 2019; Vogel et al., 2005).

We also calculated the filtering benefit, defined as the difference in accuracy between the filtering arrays and the sixtargets arrays (i.e., filtering benefit $=3 \mathrm{~T} 3 \mathrm{D}-6 \mathrm{~T}$ ). Higher scores indicate that introducing distractors that need to be ignored improves performance, relative to performance with a large-size array with targets only. The benefit score is not commonly used in the literature on filtering in VWM. This is because participants that exhibit a capacity of three or above already with large, six-target arrays are not likely to improve when three out of the six items are designated as distractors (in the 3T3D array), simply because three is the highest possible capacity score in that latter condition. For that reason, we followed up our overall analyses of filtering benefit by looking specifically at low-capacity individuals, for whom improvement would be viable.

\section{Method}

\section{Participants}

Sixty-nine Tel-Aviv University students (51 women, 18 men; $M_{\text {age }}=24.49$ years, $S D=3.18$ ) were paid 40 NIS (around $\$ 10$ at the time of the experiment) for participation. One participant was excluded due to below-chance performance on the task. All participants reported normal or corrected-tonormal visual acuity and normal color vision.

\section{Materials}

Mindset manipulation We used the mindset induction manipulation developed by Freitas et al. (2004). In the abstract mindset condition, participants were presented with five vertically aligned boxes connected with upward arrows. In the 
first box, located at the bottom of the page, was the statement: "Maintain good physical health." Participants were instructed to answer Why? in each of the four consecutive boxes. For example, a possible answer could have been "to feel better." The next box would then prompt participants to ask themselves, "Why do I want to feel better?" In this way, participants provided four recursive "why" responses. In the concrete mindset condition, participants were also presented with five vertically aligned boxes, but this time the first box, with the statement "Maintain good physical health," was located at the top of the page, and participants were instructed to answer How? in each of the four consecutive boxes, which were connected with downward arrows. After providing an answer, participants indicated how they would perform their previous action. For example, participants could have responded to the question by answering "lose weight." In the following box, they would then have to answer the question "How would you lose weight?," and so on (see Fig. 1).

Filtering task We used a variant of the change-detection task that directly investigates filtering efficiency (Allon \& Luria, 2017, 2019; Vogel et al., 2005). Each trial in the task started with the presentation of a black fixation sign ("+") in the middle of the screen for $500 \mathrm{~ms}$. Then a display of either three targets, six targets, or three targets and three distractors (i.e., the filtering condition) was presented, in intermixed random order. Colored pacman shapes served as the targets, and circles served as the distractors (see Fig. 1). The color of each stimulus was randomly selected, with no repetition per array,

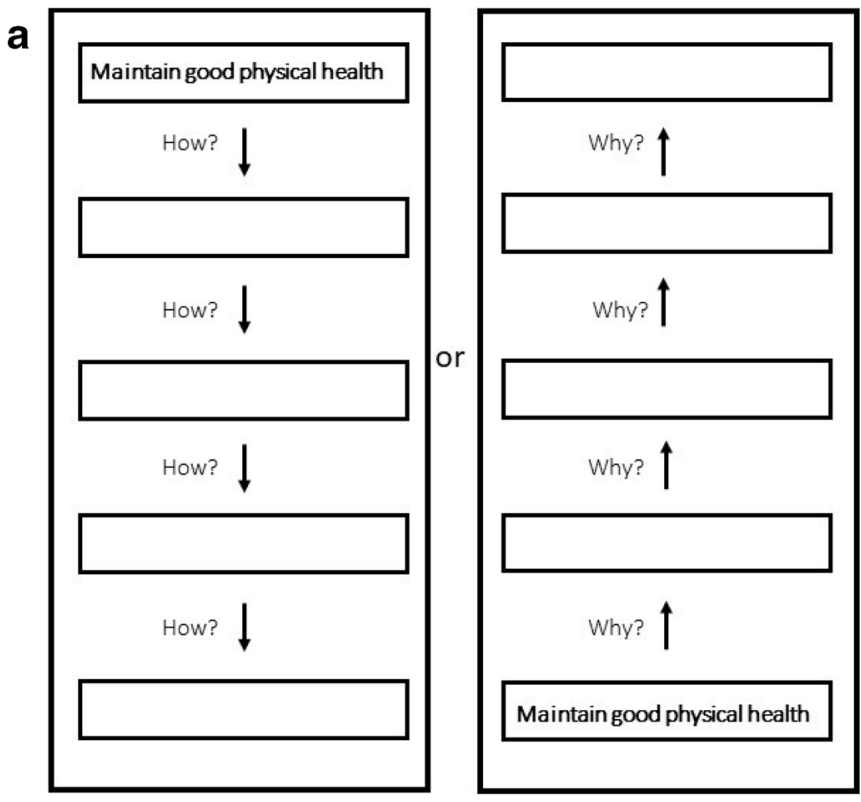

Fig. 1 (a) Illustration of the mindset manipulation task. In different blocks, participants answered four consecutive questions on either "how" or "why" to maintain good physical health and then proceeded to the filtering task (b), in which a sample array was followed by a blank from a set of eight colors — black, blue, cyan, green, pink, red, white, and yellow - and was presented on a gray background. All stimuli were randomly positioned within a $18^{\circ} \times 18^{\circ} \mathrm{re}-$ gion on the monitor, with the constraint that the minimal distance between each two stimuli was at least $2.7^{\circ}$ of visual angle (center to center). The stimulus radius was about $0.75^{\circ}$ of visual angle from a seated distance of approximately $60 \mathrm{~cm}$. On trials in which the test probe was different from the item in the original array, the changed item was replaced with a color not presented in the sample array. Stimuli were presented for $200 \mathrm{~ms}$ and were followed by a retention interval of $900 \mathrm{~ms}$, during which a blank screen was presented. A test probe then appeared in one of the pervious target locations, and participants indicated whether the test probe had the same color as or a different color from the target color that had appeared at that location.

The filtering cost was calculated as the difference in accuracy between the three-target array and the filtering array (filtering cost $=3 \mathrm{~T}-3 \mathrm{~T} 3 \mathrm{D}$ ). A score near zero would indicate perfect filtering, such that performance was not affected by distractors. A higher score, in contrast, would indicate that distractors interfered with performance. The filtering benefit was calculated as the difference in accuracy between the filtering array and the six-target array (filtering benefit $=3 \mathrm{~T} 3 \mathrm{D}-$ 6T). A higher score would indicate better performance when some items in an array were designated as distractors than for a similar-sized array with targets only. A score near zero would indicate no advantage to designating some of the stimuli in an array as distractors.

b

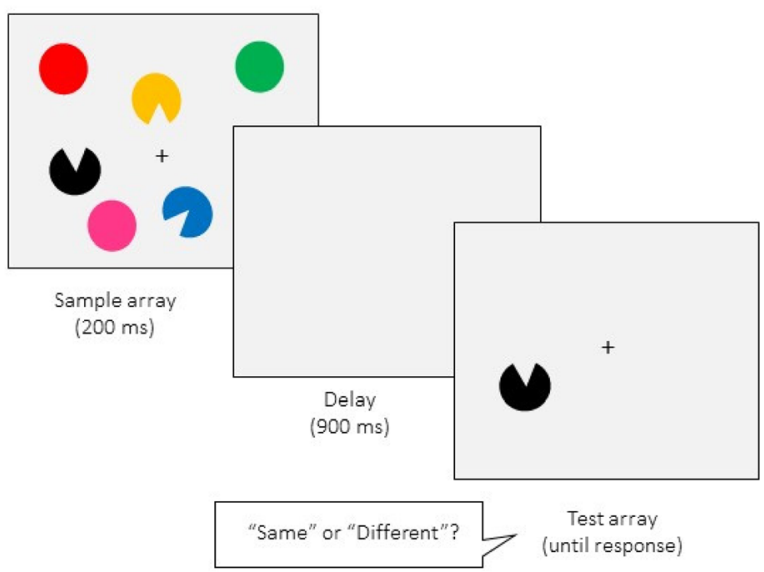

delay and then a test array. The arrays contained either three targets, six targets, or three targets and three distractors (shown here). Participants were asked to ignore the distractors (circles) and to indicate whether a change was present in one of the targets (pacman shapes) 


\section{Procedure}

Each participant completed the filtering task under three conditions: without any manipulation (i.e., baseline condition), and under concrete and abstract mindsets. To prevent any possible carryover effects from the mindset manipulations, the baseline condition was always done first, but the order of the latter two blocks was counterbalanced between participants. In this way, the comparison between abstract and concrete mindsets did not confound order, but the comparison of each of these conditions to the baseline did. In view of this (and because order effects did emerge), we analyzed the results both with and without the baseline condition. Overall, participants performed 360 critical trials, in three blocks of 120 trials each.

\section{Results and discussion}

\section{Accuracy}

Accuracy was submitted to a 2 (order: abstract block first vs. concrete block first) $\times 3$ (mindset: abstract, concrete, baseline) $\times 3$ (array: 3T, 6T, 3T3D) mixed-design analysis of variance (ANOVA), with only order manipulated between participants. The analysis revealed a main effect of mindset, $F(2,134)=$ $8.77, p<.001, \eta_{\mathrm{p}}{ }^{2}=.116$, and a main effect of array, $F(2,134)$ $=274.91, p<.001, \eta_{\mathrm{p}}{ }^{2}=.804$. The effect of order was not significant, $F<1$, and neither the interaction of order and mindset nor the interaction of order, mindset, and array reached significance, $F<1$. The interaction of order and array was marginally significant, $F(2,134)=2.813, p=.064, \eta_{\mathrm{p}}{ }^{2}=$
.040. Most importantly, a significant interaction between mindset and array, $F(4,268)=2.870, p=.031, \eta_{\mathrm{p}}{ }^{2}=.039$, suggested that the filtering cost and filtering benefit both depended on mindset (Fig. 2). To examine this interaction, and more directly to test our prediction, we compared the filtering cost and filtering benefit between abstract and concrete mindsets.

\section{Filtering cost}

To test our central prediction about the effect of abstract versus concrete mindsets on filtering efficiency, filtering costs were submitted to 2 (mindset: abstract vs. concrete) $\times 2$ (order: abstract block first vs. concrete block first) mixed-design ANOVA. The predicted effect of mindset emerged, whereby the filtering cost was higher in the concrete mindset condition $(M=.171, S D=.128)$ than in the abstract mindset condition $(M=.141, S D=.091), F(1,67)=5.17, p=.026, \eta_{\mathrm{p}}{ }^{2}=.072 . \mathrm{A}$ main effect of order, $F(1,67)=3.86, p=.054, \eta_{\mathrm{p}}{ }^{2}=.054$, indicated that the filtering cost was higher for participants who completed the abstract mindset condition first than for those who completed the concrete mindset condition first. The interaction of order and mindset was not significant, $F<1$, indicating that the effect of abstraction on filtering cost was obtained irrespective of order.

\section{Filtering benefit}

Filtering benefit scores were submitted to the same 2 (mindset: abstract vs. concrete) $\times 2$ (order: abstract block first vs. concrete block first) mixed-design ANOVA. The predicted effect

1.00

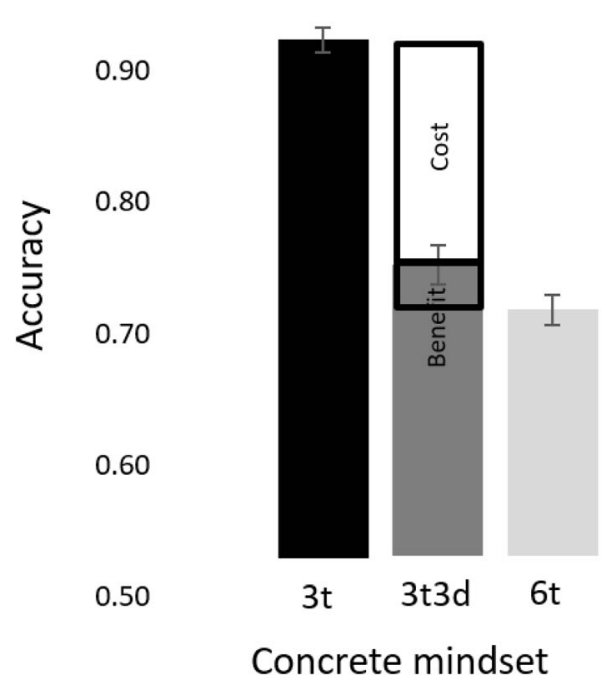

Fig. 2 Accuracy by type of array and mindset. Filtering cost is calculated as the difference between the three-targets condition (3t) and the filtering condition (3t3d). Filtering benefit is calculated as the difference between

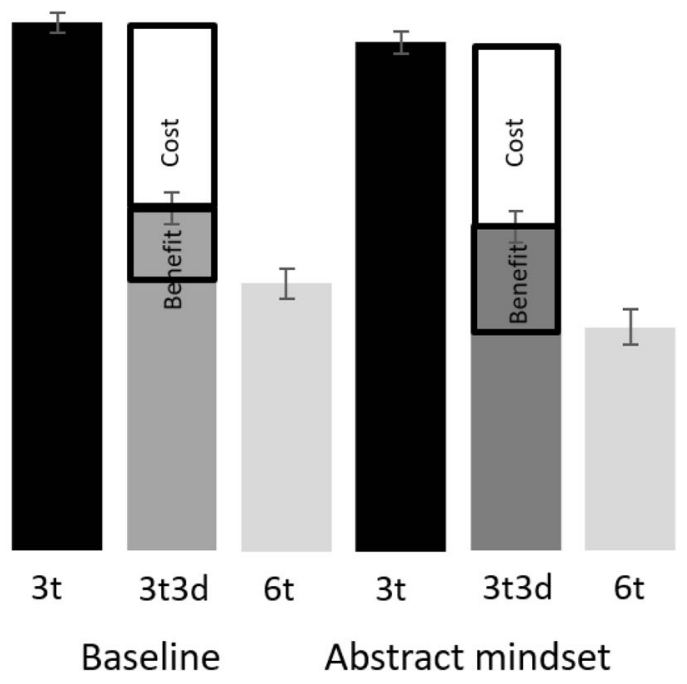

the filtering condition ( $3 \mathrm{t} 3 \mathrm{~d})$ and the six-targets condition (6t). Bars indicate standard errors 
of mindset emerged, whereby filtering benefit was lower in the concrete mindset condition $(M=.034, S D=.100)$ than in the abstract mindset condition $(M=.076, S D=.118), F(1,67)$ $=5.17, p=.005, \eta_{\mathrm{p}}{ }^{2}=.114$. Neither order nor the interaction of order and mindset was significant, $F<1$.

As we noted before, benefit scores are constrained, such that if one can recall three items or more in the 6T array, then he or she is even more likely to do so with the "help" of the three to-be-ignored distractors in the 3T3D array. We therefore conducted the same analysis for participants with a capacity of 2.5 or fewer items in the 6T array $(n=38)$. Within this group, benefit scores were submitted to mixed-design ANOVA, with mindset as a within-participants factor and order as a betweenparticipants factor. As predicted, an effect of mindset indicated that the filtering benefit was lower in the concrete mindset condition $(M=.049, S D=.105)$ than in the abstract mindset condition $(M=.121, S D=.112), F(1,36)=17.12, p<.0001$, $\eta_{\mathrm{p}}{ }^{2}=.322$. The same analysis with participants whose capacity in the 6T arrays was higher than 2.5 items $(n=31)$ did not show an effect of mindset, $F<1$.

\section{Comparison to the baseline condition}

As we noted earlier, the order of the baseline condition could not be counterbalanced with the other conditions, but rather always preceded them. Thus, comparisons of the experimental conditions to the baseline, although informative, should be interpreted with caution. Planned comparisons revealed that the filtering cost in the baseline condition $(M=.141, S D=$ $.098)$ was not different from that in the abstract condition $(M=$ $.141, S D=.091), F<1$, and was significantly lower than that in the concrete condition $(M=.171, S D=.128), F(1,67)=$ $5.23, p=.025, \eta_{\mathrm{p}}{ }^{2}=.071$. The filtering benefit in the baseline condition $(M=.057, S D=.099)$ was not significantly different from that in the abstract condition $(M=.076, S D=.118), F(1$, $68)=1.063, p=.306$, and was marginally higher than that in the concrete condition $(M=.034, S D=.100), F(1,68)=$ $3.163, p=.08, \eta_{\mathrm{p}}{ }^{2}=.044$. Thus, it seems that a concrete mindset hindered filtering relative to the baseline, whereas the abstract mindset did not improve filtering relative to the baseline.

\section{Study 2}

Study 2 was designed to replicate Study 1 with several changes. First, we used the same mindset manipulation with different operationalization in order to enhance generalizability beyond the specific content of the manipulation in Study 1. In the present version, participants described either why they participate in experiments (abstract mindset) or how to participate in experiments (concrete mindset). A similar pattern of results in Study 2 would be more easily attributable to the mindset manipulation than to any specific content that the manipulation might have elicited. Second, to avoid any effects of order, we decided to adopt a between-participants design. The same changedetection task was administered as in Study 1.

\section{Method}

\section{Participants}

One hundred Tel-Aviv University students (79 women, 21 men; $M_{\text {age }}=22.86$ years, $S D=1.82$ ) participated in the study for course credits. Two participants were excluded: one for not completing the manipulation task, and one for failing to complete the memory task. All participants reported normal or corrected-to-normal visual acuity and normal color vision.

\section{Materials}

Mindset manipulation We used the same mindset manipulation as in Study 1, but instead of answering why or how one could "maintain good physical health," participants answered why or how one would "participate in experiments at the university."

\section{Procedure}

Participants were randomly assigned to the concrete or the abstract mindset manipulation. First, participants completed ten practice trials of the filtering task. Following the mindset manipulation, they completed the rest of the filtering task, which consisted of 60 trials for each array type (180 trials in total). At the end of the session, participants provided demographic information.

\section{Results and discussion}

\section{Accuracy}

Accuracy was submitted to a 3 (array: 3T, 6T, 3T3D) $\times 2$ (mindset: abstract vs. concrete) mixed-design ANOVA, with the latter variable being manipulated between participants. Not surprisingly, the analysis revealed a main effect of array, $F(2,192)=280.69, p<.001, \eta_{\mathrm{p}}{ }^{2}=.745$. The effect of mindset was not significant, $F(1,96)=.968, p=.328, \eta_{\mathrm{p}}{ }^{2}=.01$, and the interaction of array and mindset was marginally significant, $F(2,192)=2.43, p=.090, \eta_{\mathrm{p}}{ }^{2}=.025$, suggesting that filtering efficiency depended to some extent on mindset. To more closely examine our main predictions, we compared the filtering cost and filtering benefit between abstract and concrete mindsets (see Fig. 3). 
1.00

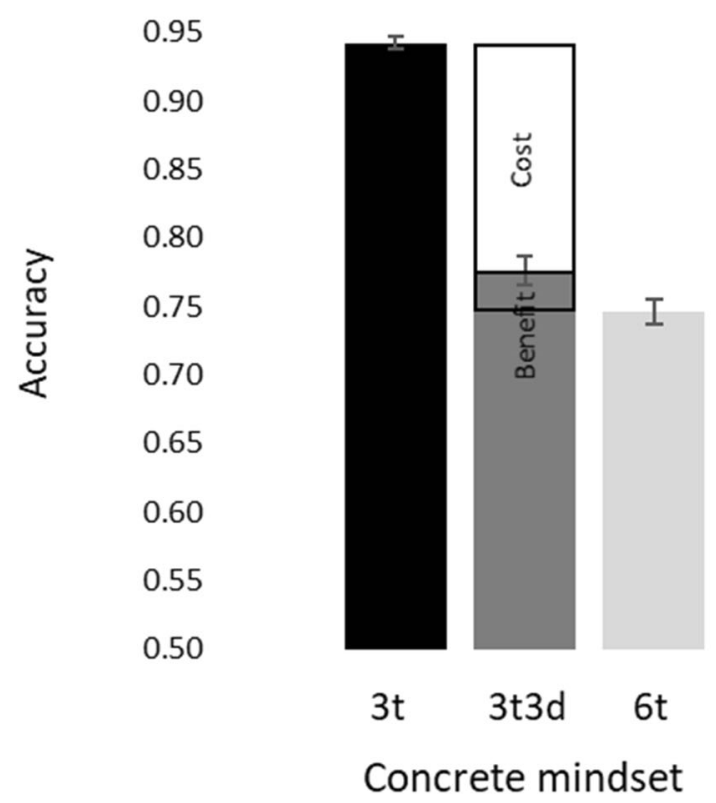

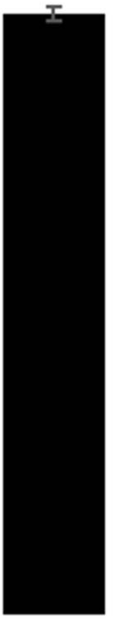
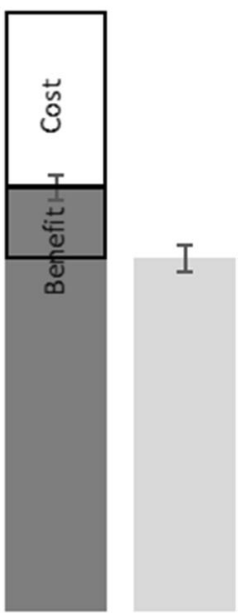

$3 t \quad 3 t 3 d$

$6 \mathrm{t}$
Concrete mindset

Fig. 3 Accuracy by type of array and mindset. Filtering cost is calculated as the difference between the three-targets condition ( $3 \mathrm{t}$ ) and the filtering condition ( $3 \mathrm{t} 3 \mathrm{~d})$. Filtering benefit is calculated as the difference between

\section{Filtering cost}

Filtering cost scores were submitted to a one-way ANOVA with mindset (concrete vs. abstract) as a between-participants factor. The predicted effect of mindset emerged, whereby filtering cost was higher in the concrete mindset condition $(M=$ .166, $S D=.093)$ than in the abstract mindset condition $(M=$ $.130, S D=.081), F(1,96)=4.20, p=.043, \eta_{\mathrm{p}}^{2}=.042$.

\section{Filtering benefit}

Filtering benefit scores were submitted to the same one-way ANOVA, with mindset (concrete vs. abstract) as a betweenparticipants factor. However, contrary to our prediction, mindset did not significantly influence filtering benefit, $F(1$, 96) $=1.50, p=.224, \eta_{\mathrm{p}}{ }^{2}=.015$.

As we noted in Study 1, filtering can be expected to benefit the performance of participants whose capacity in $6 \mathrm{~T}$ arrays is lower than 3 items. Therefore, we conducted the same analysis on participants whose capacity was 2.5 or fewer items in the $6 \mathrm{~T}$ arrays of the abstract and concrete conditions combined ( $n$ $=30$ ). As predicted, the effect of mindset emerged, whereby filtering benefit was lower in the concrete mindset condition $(M=.047, S D=.093)$ than in the abstract mindset condition $(M=.132, S D=.059), F(1,28)=8.796, p=.006, \eta_{\mathrm{p}}{ }^{2}=.239$. The same analysis with participants whose capacity in the 6T arrays was higher than $2.5(n=68)$ did not yield an effect of mindset, $F<1$. the filtering condition (3t3d) and the six-targets condition (6t). Bars indicate standard errors

\section{Abstract mindset}

\section{General discussion}

In two studies, we induced in participants concrete and abstract mindsets and examined performance in a visual working memory task that required filtering of distractors in arrays that contained targets and distractors. As predicted, the concrete mindset, relative to the abstract mindset, impaired filtering efficiency. We used two measures of filtering efficiency: (a) Filtering cost, which has been used in previous studies on filtering in VWM and quantifies the extent to which adding distractors that need to be ignored interferes with performance relative to -three-target arrays. In both studies, we found a higher filtering cost in the concrete than in the abstract mindset condition. (b) Filtering benefit, quantifies the extent to which designating some items as distractors that need to be ignored improves performance relative to six-target arrays. The filtering benefit is constrained, because it is not expected for participants who can remember three or more items even in relatively large, six-target arrays (and who thus cannot be helped by designating some of the six items as distractors). For that reason, we looked at filtering benefit acores among participants with VWM capacity of 2.5 or fewer items. Among these participants, in both studies we found a lower filtering benefit in the concrete than in the abstract condition (when all participants were examined, the effect of mindset was significant only in Study 1).

In Study 1, we also included a baseline condition with no manipulation. As compared to baseline, we found impaired filtering in the concrete mindset condition, and no improved 
filtering in the abstract mindset condition. It is important to note that the baseline condition always preceded the other two mindset manipulations, and therefore the latter result should be interpreted with caution. Regardless of the question of whether the effect of mindset should be attributed to (the hindering effect of) the concrete mindset or (the facilitative effect of) the abstract mindset, the results of both studies clearly suggest that a situationally induced mindset can change filtering efficiency, even within the same person and context.

How does a concrete mindset impair filtering? According to our theoretical framework, abstraction is a process in which goal-irrelevant information is omitted while goal-relevant information is retained. For example, representing "cellular phone" as "a communication device" retains the feature "can be used to make calls" and omits the feature "can be carried in one's pocket." On this view of abstraction, an abstract representation of a visual array that contains both targets and distractors would contain only targets and omit distractors, because only the former are defined in the task as goal relevant. A concrete representation, on the other hand, is more likely to contain both targets and distractors. Being in an abstract mindset increases the tendency to use abstract representations of stimuli. In the case of composite arrays, having such a mindset means filtering out distractors.

Perhaps another mechanism is grouping. Abstraction might be viewed as a process that groups exemplars together. For example, "bread" (a more abstract category) groups together exemplars such as bagel, rye bread, and white bread. (Note that here, too, central features are retained and peripheral/ incidental features are omitted when moving to the more abstract category.) Recent studies have shown that grouping distractors together assists in filtering them out (Allon, Vixman, \& Luria, 2018). Possibly, a concrete mindset made participants view the distractors as distinct exemplars (e.g., a red packman with an upward-pointing mouth, a pink packman with a right-pointing mouth), whereas an abstract mindset made them group distractors together into a more abstract category (e.g., pacmans), which helped filtering them out.

VWM capacity theories are dominated by two different approaches: discrete slots (Zhang \& Luck, 2008) and continuous resources (Bays \& Husain, 2008; Van den Berg, Shin, Chou, George, \& Ma, 2012). Although it is still debated which approach best describes the mechanism underlying VWM storage, we argue that our results are in line with both models. The discrete slots approach assumes a limited object-based storage. When the number of objects exceeds the maximum capacity, no further information on other objects will be retained. In this case, a concrete mindset might have promoted the encoding of irrelevant distractors, consuming storage that could have been used to store target objects. An additional explanation comes from theories that conceptualize VWM as a flexible resource that dynamically adjusts the trade-off between precision of object representation and their quantity
(Bays, Catalao, \& Husain, 2009; Bays \& Husain, 2008). Although the theory is more speculative, it is possible that a concrete mindset increased precision of object representation, either target or distractor. Previous research has indeed shown that concrete representations include more details than abstract representations (for a review, see Liberman \& Trope, 2014). Representing targets in greater precision helped memory but representing distractors in greater precision did not. In fact, representing distractors in greater precision diverted resources from representing targets, and thus hindered memory for filtering arrays. As a result, a concrete (relative to an abstract) mindset reduced memory for the filtering arrays, thereby increasing filtering cost. Notably, concrete/abstract mindset can theoretically affect the representation of arrays irrespective of the exact capacity model of VWM.

Working memory capacity appears to be stable across situations, with a test-retest reliability of .8 over a period of 1.5 years (Johnson et al., 2013), and to be almost entirely immune to practice (Xu, Adam, Fang, \& Vogel, 2018). However, recent research has demonstrated that performance is affected by situational factors such as sleep deprivation (Smith, McEvoy, \& Gevins, 2002), mood (Brose, Schmiedek, Lövdén, \& Lindenberger, 2012), instruction-induced strategies (Bengson \& Luck, 2016), and expectations (Sheremata \& Shomstein, 2017). The present research supports the latter approach, by showing situational variations in WM capacity as a function of mindset.

In sum, filtering is an important cognitive process that may underlie efficient storage of information in VWM (Vogel et al., 2005). Our studies demonstrated situational effects of concrete versus abstract mindsets on filtering efficiency. These results hold promise not only to help us better understand VWM, but also to allow us to avoid situations in which VWM might be impaired, and to create situations in which VWM is expected to be optimal.

Author note B.H thanks Ayala S. Allon for her help. This research was supported by the Israel Science Foundation through Grant No. 524/17, awarded to N.L., and Grant No. $862 / 17$, awarded to R. L. All data and material are available at https://osf.io/846ey.

\section{References}

Allon, A. S., \& Luria, R. (2017). Compensation mechanisms that improve distractor filtering are short-lived. Cognition, 164, 74-86.

Allon, A. S., \& Luria, R. (2019). Filtering performance in visual working memory is improved by reducing early spatial attention to the distractors. Psychophysiology, 56, e13323. https://doi.org/10.1111/ psyp. 13323

Allon, A. S., Vixman, G., \& Luria, R. (2018). Gestalt grouping cues can improve filtering performance in visual working memory. 
Psychological Research. Advance online publication. https://doi. org/10.1007/s00426-018-1032-5

Alloway, T. P., \& Alloway, R. G. (2010). Investigating the predictive roles of working memory and IQ in academic attainment. Journal of Experimental Child Psychology, 106, 20-29.

Awh, E., Barton, B., \& Vogel, E. K. (2007). Visual working memory represents a fixed number of items regardless of complexity. Psychological Science, 18, 622-628. https://doi.org/10.1111/j. 1467-9280.2007.01949.x

Awh, E., \& Vogel, E. K. (2008). The bouncer in the brain. Nature Neuroscience, 11, 5-6.

Baddeley, A. (2003). Working memory: Looking back and looking forward. Nature Reviews Neuroscience, 4, 829-839. https://doi.org/10. 1038/nrn1201

Bays, P. M., Catalao, R. F. G., \& Husain, M. (2009). The precision of visual working memory is set by allocation of a shared resource. Journal of Vision, 9(10), 7:1-11. https://doi.org/10.1167/9.10.7

Bays, P. M., \& Husain, M. (2008). Dynamic shifts of limited working memory resources in human vision. Science, 321, 851-854. https:// doi.org/10.1126/science. 1158023

Bengson, J. J., \& Luck, S. J. (2016). Effects of strategy on visual working memory capacity. Psychonomic Bulletin \& Review, 23, 265-270.

Brose, A., Schmiedek, F., Lövdén, M., \& Lindenberger, U. (2012). Daily variability in working memory is coupled with negative affect: the role of attention and motivation. Emotion, 12, 605-617.

Burgoon, E. M., Henderson, M. D., \& Markman, A. B. (2013). There are many ways to see the forest for the trees: A tour guide for abstraction. Perspectives on Psychological Science, 8, 501-520.

Conway, A. R. A., Kane, M. J., Bunting, M. F., Hambrick, D. Z., Wilhelm, O., \& Engle, R. W. (2005). Working memory span tasks: A methodological review and user's guide. Psychonomic Bulletin \& Review, 12, 769-786. https://doi.org/10.3758/BF03196772

Conway, A. R. A., Kane, M. J., \& Engle, R. W. (2003). Working memory capacity and its relation to general intelligence. Trends in Cognitive Sciences, 7, 547-552. https://doi.org/10.1016/j.tics.2003.10.005

Cowan, N., Elliott, E. M., Saults, J. S., Morey, C. C., Mattox, S., Hismjatullina, A., \& Conway, A. R. A. (2005). On the capacity of attention: Its estimation and its role in working memory and cognitive aptitudes. Cognitive Psychology, 51, 42-100. https://doi.org/10. 1016/j.cogpsych.2004.12.001

Freitas, A. L., Gollwitzer, P., \& Trope, Y. (2004). The influence of abstract and concrete mindsets on anticipating and guiding others' selfregulatory efforts. Journal of Experimental Social Psychology, 40, 739-752.

Fujita, K., Trope, Y., Liberman, N., \& Levin-Sagi, M. (2006). Construal levels and self-control. Journal of Personality and Social Psychology, 90, 351-367.

Fukuda, K., Vogel, E., Mayr, U., \& Awh, E. (2010). Quantity, not quality: The relationship between fluid intelligence and working memory capacity. Psychonomic Bulletin \& Review, 17, 673-679. https:// doi.org/10.3758/17.5.673

Fukuda, K., \& Vogel, E. K. (2011). Individual differences in recovery time from attentional capture. Psychological Science, 22, 361-368.

Fukuda, K., Woodman, G. F., \& Vogel, E. K. (2015). Individual differences in visual working memory capacity: Contributions of attentional control to storage. In P. Jolicœur, C. Fevebvre, \& J. MartinezTrujillo (Eds.), Mechanisms of sensory working memory: Attention and performance XXV (pp. 105-119). San Diego, CA: Academic Press Elsevier.

Gaspar, J. M., Christie, G. J., Prime, D. J., Jolicœur, P., \& McDonald, J. J. (2016). Inability to suppress salient distractors predicts low visual working memory capacity. Proceedings of the National Academy of Sciences, 113, 3693-3698.

Gilead, M., Liberman, N., \& Maril, A. (2013). From mind to matter: neural correlates of abstract and concrete mindsets. Social Cognitive and Affective Neuroscience, 9, 638-645.
Greenwald, A. G. (1976). Within-subjects designs: To use or not to use? Psychological Bulletin, 83, 314-320. https://doi.org/10.1037/00332909.83.2.314

Gruber, J., Harvey, A. G., \& Johnson, S. L. (2009). Reflective and ruminative processing of positive emotional memories in bipolar disorder and healthy controls. Behaviour Research and Therapy, 47, 697704.

Johnson, M. K., McMahon, R. P., Robinson, B. M., Harvey, A. N., Hahn, B., Leonard, C. J., et al. (2013). The relationship between working memory capacity and broad measures of cognitive ability in healthy adults and people with schizophrenia. Neuropsychology, 27, 220229.

Kille, D. R., Eibach, R. P., Wood, J. V., \& Holmes, J. G. (2017). Who can't take a compliment? The role of construal level and self-esteem in accepting positive feedback from close others. Journal of Experimental Social Psychology, 68, 40-49.

Kross, E., Bruehlman-Senecal, E., Park, J., Burson, A., Dougherty, A., Shablack, H., ... Ayduk, O. (2014). Self-talk as a regulatory mechanism: How you do it matters. Journal of Personality and Social Psychology, 106, 304-324.

Liberman, N., \& Trope, Y. (2008). The psychology of transcending the here and now. Science, 322, 1201-1205.

Liberman, N., \& Trope, Y. (2014). Traversing psychological distance. Trends in Cognitive Sciences, 18, 364-369.

Luck, S. J., \& Vogel, E. K. (2013). Visual working memory capacity: From psychophysics and neurobiology to individual differences. Trends in Cognitive Sciences, 17, 391-400. https://doi.org/10. 1016/j.tics.2013.06.006

Sheremata, S. L., \& Shomstein, S. (2017). Task set induces dynamic reallocation of resources in visual short-term memory. Psychonomic Bulletin \& Review, 24, 1113-1120

Shipstead, Z., Redick, T. S., Hicks, K. L., \& Engle, R. W. (2012). The scope and control of attention as separate aspects of working memory. Memory, 20, 608-628. https://doi.org/10.1080/09658211.2012. 691519

Smith, M. E., McEvoy, L. K., \& Gevins, A. (2002). The impact of moderate sleep loss on neurophysiologic signals during workingmemory task performance. Sleep, 25, 56-66.

Trope, Y., \& Liberman, N. (2010). Construal-level theory of psychological distance. Psychological Review, 117, 440-463. https://doi.org/ 10.1037/a0018963

Van den Berg, R., Shin, H., Chou, W.-C., George, R., \& Ma, W. J. (2012) Variability in encoding precision accounts for visual short-term memory limitations. Proceedings of the National Academy of Sciences, 109, 8780-8785.

Vogel, E. K., McCollough, A. W., \& Machizawa, M. G. (2005). Neural measures reveal individual differences in controlling access to working memory. Nature, 438, 500-503. https://doi.org/10.1038/ nature 04171

Wakslak, C., \& Trope, Y. (2009). The effect of construal level on subjective probability estimates. Psychological Science, 20, 52-58.

Xu, Z., Adam, K. C. S., Fang, X., \& Vogel, E. K. (2018). The reliability and stability of visual working memory capacity. Behavior Research Methods, 50, 576-588. https://doi.org/10.3758/s13428-017-0886-6

Zhang, W., \& Luck, S. J. (2008). Discrete fixed-resolution representations in visual working memory. Nature, 453, 233-235. https://doi.org/ 10.1038/nature06860

Zinchenko, A., Conci, M., Müller, H. J., \& Geyer, T. (2018). Predictive visual search: Role of environmental regularities in the learning of context cues. Attention, Perception, \& Psychophysics, 80, 10961109. https://doi.org/10.3758/s13414-018-1500-4

Publisher's note Springer Nature remains neutral with regard to jurisdictional claims in published maps and institutional affiliations. 